\title{
A Dynamic Cooperative Scheme with Multiple Antennas for Indoor Mobile Robot Localization
}

\author{
Chung-Liang Chang and Bo-Han Wu \\ Department of Biomechatronics Engineering, National Pingtung University of Science and Technology, \\ No. 1 Shuefu Road, Neipu, Pingtung County 91201, Taiwan \\ Correspondence should be addressed to Chung-Liang Chang; chungliang@mail.npust.edu.tw
}

Received 12 June 2013; Accepted 28 August 2013

Academic Editor: Chang-Hua Lien

Copyright (C) 2013 C.-L. Chang and B.-H. Wu. This is an open access article distributed under the Creative Commons Attribution License, which permits unrestricted use, distribution, and reproduction in any medium, provided the original work is properly cited.

\begin{abstract}
This paper proposes a spatial cooperative diversity and decision-making technique to enhance signal detection and indoor mobile robot positioning performance of a global positioning satellite system (GNSS) receiver. Though the adaptive antenna array technique in early research could effectively promote antijamming freedom, overcome time-varying system, and mitigate narrowband and wideband interferences, factors such as the decrease of signal magnitude caused by obstacles (especially in the indoor environment), multipath, and blanking effect caused by the change in antenna direction with the motion of mobile robot can degrade the detection and interference mitigation performance of GNSS receivers. This paper aims to develop a dynamic cooperative scheme to proceed with the switch, selection, combination, and optimization among antennas. In addition, a signal processing experimental platform is also established to receive actually indoor GNSS signals for verification. The proposed scheme is capable of effectively promoting the postcorrelation signal-to-noise ratio (SNR) capability of a GNSS receiver under the indoor environment.
\end{abstract}

\section{Introduction}

The global navigation satellite system (GNSS) signal can be considered as a combination of signal power, carrier, spread code, and navigation message, which includes intended and unintended interferences. Once the receiver gets the navigation messages of more than four satellites, then the user position, velocity, and timing can be calculated through internal core processing of the receiver. However, how to overcome the external uncertainty factor (such as interference, and noise) is the problem concerning most of the scholars and, particularly, how to make indoor positioning efficient through the use of the receiver. Generally, the present Global Positioning System (GPS) does not function well in indoor or urban canyon multipath environments, which are regarded as difficult environments. It is hard to copy the attenuated and multipath faded signals caused by transmission around and through concrete and steel building structures.

Klukas et al. analyze the attenuation level of navigation signal through the signal reflected by different materials in indoor environment. The results demonstrate that the attenuation level of signal is between 0.5 and $23 \mathrm{~dB}$ [1]. Many scholars provide techniques to improve signal detection probability in indoor environment $[2,3]$. The most common method is so called assisted-GPS (A-GPS) [4]. This method provides positioning information from a remote server in the cellular base station through wireless network. Nevertheless, GNSS suppliers expect the indoor positioning system to operate independently without wireless network and also maintain high positioning accuracy. It is difficult to obtain high positioning precision without any assisting device (such as WiFi, UWB, and ZigBee). In the current technique, the use of multiple antennas array combination method seems promising to be able to achieve indoor positioning. Tasi et al. propose Laplacian energy distribution model to derive spatial fading function under circular antenna array [5]. This attenuation signal model is often adopted to simulate and analyze communication channel response in indoor environment.

In early communication systems, the most common method was the use of antenna array with space-time coding or cooperation to increase system capacity under fading 
environment [6]. This technique was applied to interference suppression in multiinput multioutput (MIMO) system or navigation system later $[7,8]$. In recent years, some antenna array processing techniques with intelligent and switching algorithms are proposed against the narrowband/wideband interferences, mutual coupling, and noise [9-11]. However, the application of these methods to GNSS positioning is still at initial stage.

Dietrich Jr. et al. first propose the concept of antenna array in indoor environment. This method utilizes spatial diversity characteristic for antenna array to enhance the signal-tonoise (SNR) ratio [12]. After that, Chiau and Gao not only use this technique in MIMO system to increase the channel capacity but also apply it to mobile device for future Galileo navigation system $[13,14]$. The core concept is that each antenna maintains a certain distance from other antennas, and then the independent fading signal is received from each antenna. Thus, the multiple antennas combination method can be applied in this system to increase signal detection probability and SNR.

Broumandan et al. utilize the concept of diversity technique to achieve $7 \mathrm{~dB}$ signal processing gain for mobile navigation device in indoor environment [15].Zaheri et al. propose spatial diversity technique using two antennas, which results in $5 \mathrm{~dB}$ diversity gain enhancement and effectively suppresses multipath fading effect [16]. This paper proposes dynamic cooperative scheme based on the concept of antenna diversity characteristics. Through the antenna combination and decision logic process, the proposed scheme improves the signal detection probability under indoor environment. Unlike the diversity method proposed earlier $[17,18]$, this paper provides a dynamic switching and selection process for antenna array system. The experiments are also conducted with a mobile robot in indoor environment, and analysis and evaluation are demonstrated in this paper.

\section{Methodology}

The goal of antenna diversity technique is to mitigate the fading and improve the overall quality of the radio link. The basic principle is that the receiver combines more than one copy of a satellite signal where each copy is received through a different branch. As for GNSS applications, the receiver must be able to detect a weak navigation signal with -140 to $-160 \mathrm{dBm}$ power strength under outdoor environment [19]. Once the signal is masked or blanked, it is difficult for the receiver to position the user location due to signal attenuation. Thus, some diversity methods, including frequency diversity, time diversity, and antenna diversity, are proposed to solve this problem. In the following section, the dynamic cooperative scheme is demonstrated as a measure of effectiveness for multiple antennas.

2.1. Signal Model. The proposed scheme utilizes different types of antenna array combination to form a specific spatial relationship, which results in the success of navigation signal acquisition. The system model is considered to describe the relationship between each antenna location and reference point in the space. Figure 1 depicts the orientation

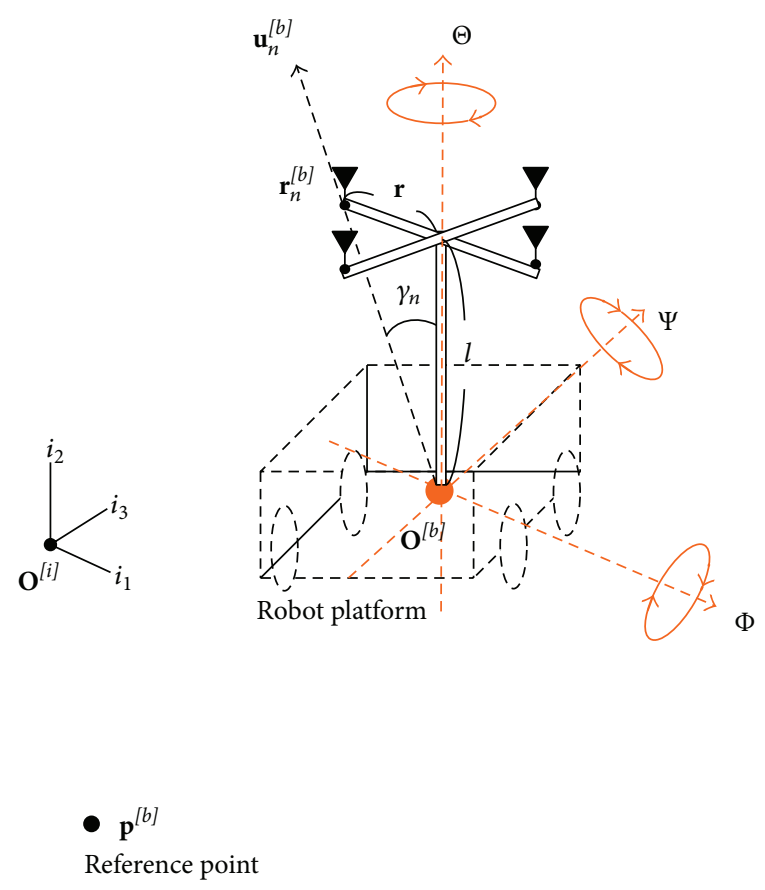

FIGURE 1: Definition of coordinate systems and locations of antenna elements.

of the robot, which is described in terms of Euler angles. Let $\mathbf{p}^{[b]}$ and $\mathbf{p}^{[e]}$ be the positions vectors in the body-fixed frame ( $b$-frame) and earth-centered earth-fixed frame ( $e$ frame), respectively. Meanwhile, let $\mathbf{O}^{[b]}$ and $\mathbf{O}^{[i]}$ be the orientation positions in the body-fixed frame ( $b$-frame) and earth-centered inertial frame ( $i$-frame), respectively. The transformation matrix $\mathbf{C}_{i}^{b}=R\left(i_{1}, \Phi\right) R\left(i_{2}, \Theta\right) R\left(i_{3}, \Psi\right)$ can be utilized to transfer the $i$-frame to the $b$-frame system. Thus, the positions vectors are $\mathbf{p}^{[b]}=\mathbf{C}_{i}^{b} \mathbf{C}_{e}^{i} \mathbf{p}^{[e]}$, where $\mathbf{C}_{e}^{i}$ represents the transformation matrix from the $e$-frame to the $i$-frame. As the robot moves, both the antenna position and the rotation matrix are also changed accordingly [20]. The location of each antenna element in the body-fixed frame is assumed to be at $\mathbf{r}_{n}^{[b]}=\left[\begin{array}{llll}l & r \cos \gamma_{n} & r \sin \gamma_{n}\end{array}\right]^{T}$, and the pointing angle of the antenna is along $\mathbf{u}_{n}^{[b]}=\left[\begin{array}{lll}0 & \cos \gamma_{n} & \sin \gamma_{n}\end{array}\right]^{T}$, where $l$ is the lever arm from the center of the gravity of the vehicle to the center of the antenna, $\gamma_{n}$ is the angle of the $n$th antenna initial location, and $r$ is the radius length. $[\cdot]^{T}$ depicts the transpose operator. Thus, assume the received signal $s_{0}(t)$ from the reference point under indoor environment can be described as follows:

$$
s_{0}(t)=\sqrt{P_{i}} g_{i}\left(t-\tau_{i, 0}\right) b_{i}\left(t-\tau_{i, 0}\right) \exp \left(j 2 \pi f_{i} t+\psi_{i}\right),
$$

where $P_{i}$ denotes the reception power of the $i$ th satellite signal, $\tau_{i, 0}$ are the delay time from the $i$ th satellite to the reference point, $b_{i}(t)$ represents the navigation data bit, $g_{i}(t)$ is the spread code sequence, $f_{i}$ is the carrier frequency, $\psi_{i}$ is the carrier phase offset, and $j=\sqrt{-1}$. Suppose that there are $N$ antenna elements employed for the reception and processing 


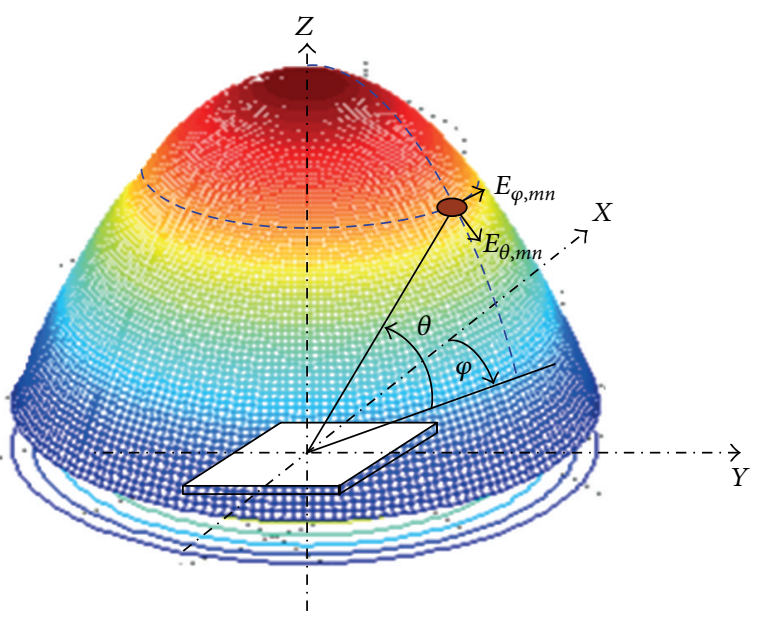

(a)

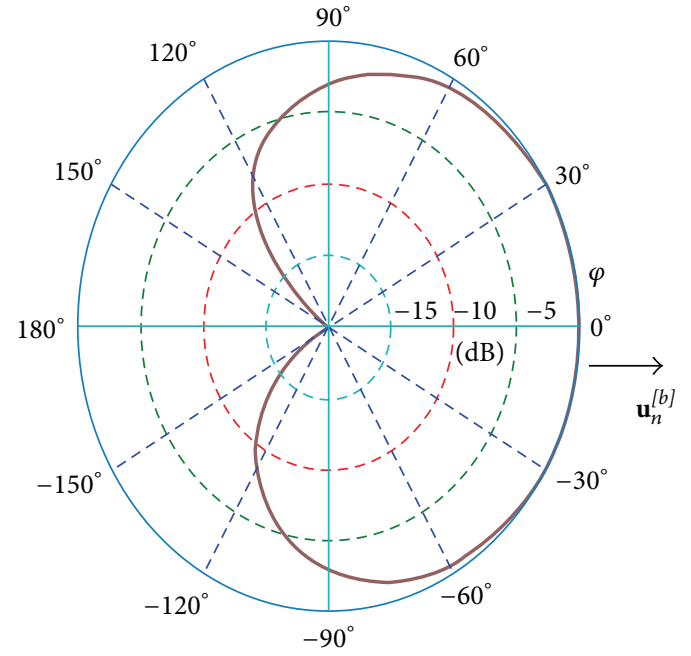

(b)

FIGURE 2: (a) The relation of angular coordinates to Cartesian coordinates. (b) Antenna gain pattern.

of GNSS signals. Then the $n$th received signal $s_{0 n}(t)$ can be described as the signal $s_{0}(t)$ with a propagation time delay:

$$
\begin{aligned}
s_{0 n}(t) & =s_{0}\left(t-\tau_{i, n}\right)+\sum_{k=1, k \neq i}^{U} s_{k}\left(t-\tau_{k, n}\right) \\
& =a_{i, n} s_{0}(t) a_{i, n}+\sum_{k=1, k \neq i}^{U} a_{k, n} s_{k, n}(t), \\
& i \in\{1,2, \ldots, U\}, n \in\{1,2, \ldots, N\},
\end{aligned}
$$

where $\tau_{i, n}=2 \pi d_{i, n} / \mathrm{c}$ depicts the propagation time delay from the $i$ th satellite signal to the reference point. The value $\tau_{i, n}$ can be transferred to phase delay. Thus, let $a_{i, n}=$ $\exp \left(-j\left(2 \pi d_{i, n} / \lambda\right) t\right)$ and $d_{i, n}=\left\langle r_{n}^{[e]}, h_{l, i}^{[e]}\right\rangle$, where $\langle *, \circ\rangle$ depicts the inner product between "*" and "o". The unit vector $h_{l, i}^{[e]}$ is from the reference point to the $i$ th incoming satellite signal, coordinated in the earth-centered earth-fixed frame (e-frame), and $c$ is the speed of light in $\mathrm{m} / \mathrm{sec} . U$ is the number of the visible satellites in the sky. Under the signal attenuation environment, each satellite signal is received by the receiver antenna from different directions with a certain angular spread $(\theta, \varphi)$ in space. The antenna correlation gain $A_{m n}(\theta, \varphi)$ and direction gain of weight factor $\delta_{n}(\theta, \varphi)$ decide the degree of the signal attenuation. The symbols $m$ and $n$ depict the $m$ th and $n$th antennas, respectively. In addition, the phase delay factor $\exp (j \bar{\psi})$ caused by multipath effect is also considered. Thus, the signal model in the fading environment is as follows:

$$
\bar{s}_{0 n}(t)=G_{n}(\theta, \varphi) \delta_{n}(\theta, \varphi) s_{0 n}(t)+w_{n}(t),
$$

where $G_{n}=A_{m n}(\theta, \varphi) \exp (j \bar{\psi})$ and $w_{n}(t)$ depicts noise term with Gaussian distribution, such as thermal noise and hardware noise.
2.2. Prediversity Process. The detection performance of weak indoor navigation signal can be enhanced through multiple antennas combination method, such as polarization diversity, spatial diversity, and pattern diversity.

Polarization diversity method combines pairs of antennas with orthogonal polarizations, which can immunize a system from polarization mismatches that would otherwise cause signal fade. The antenna correlation can be depicted as complex and envelope correlations. The envelope correlation coefficient is related to the complex correlation $\Psi_{m n}$ as shown below [21]:

$\Psi_{m n}^{2}=\frac{\int_{0}^{2 \pi} \int_{0}^{\pi} A_{m n}^{2}(\theta, \varphi) \sin ^{2} \theta d \theta d \varphi}{\int_{0}^{2 \pi} \int_{0}^{\pi} A_{m m}(\theta, \varphi) \sin \theta d \theta d \varphi \int_{0}^{2 \pi} \int_{0}^{\pi} A_{n n}(\theta, \varphi) \sin \theta d \theta d \varphi}$,

$m \neq n$,

where $A_{m n}=C \cdot E_{\theta, m n}(\theta, \varphi) P_{\theta}(\theta, \varphi)+E_{\varphi, m n}(\theta, \varphi) P_{\varphi}(\theta, \varphi)$, $E_{\theta, m n}$, and $E_{\varphi, m n}$ denotes the electric far field of the antenna from source $(\theta, \varphi)[22] . C=P_{V} / P_{H}$ is the ratio of the averaged vertical power $P_{V}$ to time averaged horizontal power $P_{H}$ in the fading environment in linear form. $P_{\varphi}(\theta, \varphi)$ and $P_{\theta}(\theta, \varphi)$ are the azimuthal angular $\varphi$ and elevation angular $\theta$ density functions of the incoming plane waves. In general, a uniform distribution is a reasonable assumption for the azimuthal angular density function as assumed earlier [23], when user of a mobile terminal moves along a random route. The angular density functions in the elevation direction are not uniformly distributed. Figure 2 shows the electric far field of the antenna.

In (4), $\Psi_{m n}^{2}=0$ is for ideal case. If the correlation coefficient is greater than zero $\left(\Psi_{m n}^{2}>0\right)$, then the diversity gain will be reduced. The analysis shows that, when the correlation is not too close to unity or $\Psi_{m n}^{2} \leq 0.7$, the degradation of 


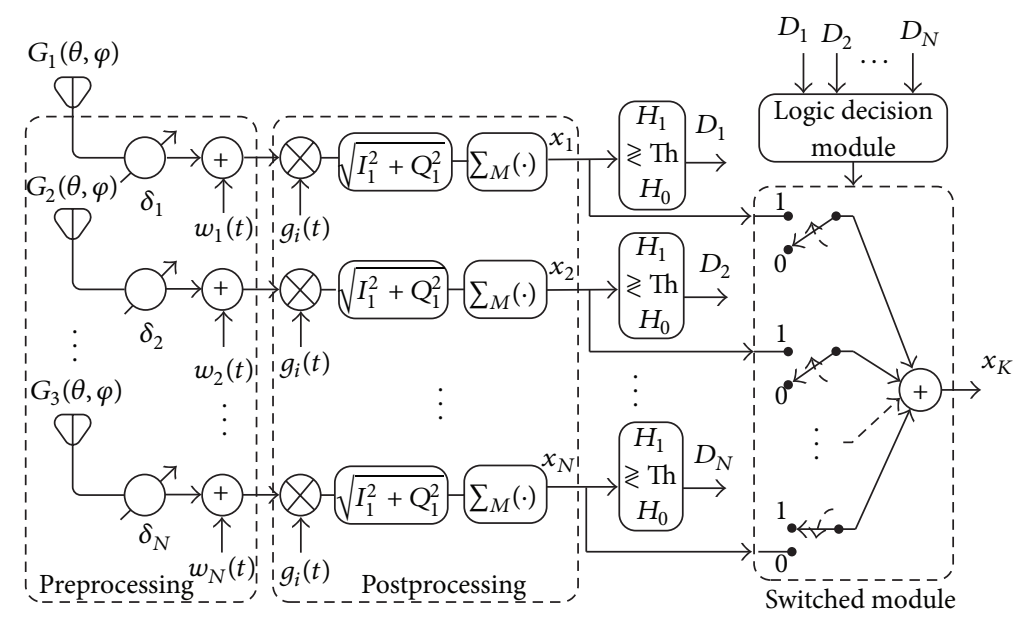

FIgURE 3: Cooperative antenna array system.

the diversity gain due to envelope correlation is given by the gain degradation factor (GDF) of the following equation:

$$
\mathrm{GDF}=\sqrt{1-\Psi_{m n}^{2}} .
$$

It is suitable to adjust the antenna spacing and the number of antennas to achieve access to the independent fading signal by using the diversity scheme. However, smaller antenna spacing will result in mutual coupling effect and degrade the effectiveness of the spatial diversity. Under the optimal antenna spacing arrangement, the use of pattern diversity method achieves different pattern shapes and thus is conducive to access a particular direction of the signal [24, 25]. The antennas serve as parasitic elements to each other and alter their patterns to allow signals to be picked up in different directions. This technique is so called "beamforming." Assume the complex weight vector $\mathbf{w}=\left[\begin{array}{llll}\delta_{1} & \delta_{2} & \cdots & \delta_{N}\end{array}\right]$ with unit norm $\left\|\mathbf{w}^{H} \mathbf{w}\right\|_{1}=1$; then the gain of antenna system is shown as follows:

$$
L=\left|\sum_{n=1}^{N} \delta_{n} G_{n}\right|^{2}=\mathbf{w}^{H} \mathbf{R}_{\mathbf{G}} \mathbf{w},
$$

where channel gain $G_{n}$ is the same as in (3) and $\mathbf{R}_{\mathbf{G}}$ is the convariance matrix of the channel response vector $\mathbf{G}=$ $\left[\begin{array}{llll}G_{1} & G_{2} & \cdots & G_{N}\end{array}\right]$. Assuming independent fading environment, $\mathbf{R}_{\mathbf{G}}=\mathbf{I}$, then array gain is equal to $1(L=1)$, which depicts the array providing no gain over a single antenna. If the fading is fully correlated between the antennas, the matrix $\mathbf{R}_{\mathbf{G}}=E\left[\mathbf{G G}^{H}\right]$ and $\mathbf{w}=\mathbf{G} /\|\mathbf{G}\|$, where $E[\cdot]$ denotes expected operator. Thus, the gain of antenna system is $N\left(L=\|\mathbf{G}\|^{2}=\right.$ $N)$.

2.3. Postdiversity Process. After obtaining independent fading paths, the next criterion in diversity technique is antenna combinations. The signals received at each branch can be combined in different ways to mitigate the effects of fading. If the two signals are uncorrelated, it is rare that the two fading signals will be in a deep null at the same time.
Thus, the combined signal creates a higher postcorrelation SNR (post-SNR) at the output compared to a single branch resulting in a diversity gain.

2.3.1. Multiple Antennas Combination Methods. It is necessary to synchronize the clock timings for each branch before using the antenna diversity technique. The decision module relies on the difference in the signal detection probability of each branch and performs the antenna selection, switch, and combination. Figure 3 depicts the system diagram including preprocessing module, postprocessing module, switched module, and logic decision module. Among them, the mode selection is built in logic decision module, which can perform different antenna combinations.

This proposed scheme can combine the received signal of each antenna through different methods, such as switched combination (SWC), selection combination (SC), equal gain combination (EGC), and maximum ratio combination (MRC) $[26,27]$.

The concept of SC method is that the rear of each antenna is equipped with a radio frequency (RF) front-end module. Each module calculates the SNR of its corresponding received signal and then selects the highest SNR as system output [27]. The SWC method quite resembles the selection method, but it is different in the priori setting of signal to noise ratio threshold employed in this method. If the SNR of selected antenna is below threshold value, then the system will switch to another antenna. The drawback of SWC is that the SNR of switched antenna may not be the highest one among them. The SC and SWC methods can only have one antenna for system output; and remaining antennas are not used. To effectively make use of all antennas and enhance signal detection performance, this method sums the received data of all the antennas to output. However, the summed signal may appear incoherent due to the inconsistent signal phase of each antenna. Thus, the rear of each antenna is multiplied by a phase compensator to render the received signal phase coherent. EGC is a special case of the maximum ratio combination. The antenna weights are set as one. 
Consequently, there is no need to adjust the weight of the individual signals before adding them, which indicates that all the antenna signals are weighed equally in spite of their SNRs. Thus, the total SNR applied to the detector after the diversity reception of $K$ selected channels is given by

$$
\rho_{K}=\frac{x_{K}^{2}}{2 \sigma_{K}^{2}}=\frac{x_{K}^{2}}{2 W_{n} K},
$$

where $\sigma_{K}^{2}=W_{n} \sum_{n=1}^{K} \delta_{n}=W_{n} K$ represents the total noise power expressed as the weighted sum of the noise powers of all received signals in the diversity reception. $W_{n}$ is the average noise power at each branch.

As for EGC, $x_{K}^{2}=\left(\sum_{n=1}^{K} x_{n}\right)^{2}=\sum_{m, n=1}^{K} \overline{x_{m} x_{n}}$, the average $\mathrm{SNR}$ is thus given by

$$
\bar{\rho}_{K}=\frac{\sum_{m, n=1}^{K} \overline{x_{m} x_{n}}}{2 W_{n} K} .
$$

Assume the noise in each of the branches is independent of noise power $\sigma^{2}$. Under Rayleigh fading channels, when $m=$ $n$,

$$
\begin{aligned}
\overline{x_{m} x_{n}} & =\overline{x_{n}^{2}}=\operatorname{var}\left(x_{n}\right)+\left(\bar{x}_{n}^{2}\right) \\
& =\left(2-\left(\frac{\pi}{2}\right)\right) \sigma^{2}+\bar{x}_{n} \cdot \bar{x}_{n} \\
& =\left(2-\left(\frac{\pi}{2}\right)\right) \sigma^{2}+\frac{\pi \sigma^{2}}{2}=2 \sigma^{2},
\end{aligned}
$$

where $\operatorname{var}(\cdot)$ is the variance operator. Under $m \neq n$, assuming that each of the diversity branches receives uncorrelated fading signal, one gets

$$
\begin{aligned}
\overline{x_{m} x_{n}} & =\bar{x}_{m} \cdot \bar{x}_{n} \\
& =\sqrt{\frac{\pi \sigma^{2}}{2}} \cdot \sqrt{\frac{\pi \sigma^{2}}{2}}=\frac{\pi \sigma^{2}}{2} .
\end{aligned}
$$

Thus, the average output SNR in (8) becomes

$$
\begin{aligned}
\bar{\rho}_{K} & =\frac{\sum_{m, n=1}^{K} \overline{y_{m} y_{n}}}{2 W_{n} K}=\frac{1}{2 W_{n} K}\left[2 K \sigma^{2}+K(K-1) \frac{\pi \sigma^{2}}{2}\right] \\
& =\kappa\left[1+(K-1) \frac{\pi}{4}\right],
\end{aligned}
$$

where $\kappa=\sigma^{2} / W_{n}$ depicting the average SNR per branch of diversity. In fact, EGC is an exception of maximum ratio combination. The received signal of each antenna module is processed through weighting, phase compensation, and sum. The reason for weighting is because the received SNR of each antenna is not the same. Low SNR of certain antennas will degrade the signal detection performance of the whole system. Thus, each signal is processed through weighting prior to phase compensation in order to maximize the SNR of the whole system. A diversity antenna with a lower SNR is given a lower weighting, and a diversity antenna with a higher SNR is given a higher weighting. In EGC, the weighting for all is set as 1 . Thus, (7) is shown as

$$
\rho_{K}=\frac{\left(x_{K} / \sqrt{2}\right)^{2}}{\sigma_{K}^{2}}=\frac{x_{K}^{2}}{2 \sigma_{K}^{2}},
$$

where $x_{K}=\sum_{n=1}^{K} \delta_{n} x_{n}$ is the envelope that is applied to the detector. $x_{n}$ is the desired signal at the $n$th antenna, and $\delta_{n}$ is the weight of the $n$th antenna. Rappaport showed that the maximum SNR of the detector is expressed as [7]

$$
\begin{aligned}
\rho_{K} & =\frac{x_{K}^{2}}{2 \sigma_{K}^{2}}=\frac{1}{2 W_{n}} \cdot \frac{\left(\sum_{n=1}^{K}\left(x_{n}^{2} / W_{n}\right)\right)^{2}}{\sum_{n=1}^{K}\left(x_{n}^{2} /\left(W_{n}\right)^{2}\right)} \\
& =\sum_{n=1}^{K} \frac{x_{n}^{2}}{2 W_{n}}=\sum_{n=1}^{K} \rho_{n},
\end{aligned}
$$

where $\rho_{n}$ and $W_{n}$ indicate the SNR and the average noise power at the $n$th antenna, respectively. Thus, it can be said from (13) that the output SNR applied to the detector utilizing $K$ selected branches equals the sum of SNR of various antenna signals. The probability density function of the output $\operatorname{SNR} \rho_{K}$ can be shown as a chi-square random variable with $2 K$ degree of freedom with average SNR being equal to $\sigma^{2} / 2 W_{n}=\kappa / 2$, and it can be demonstrated as [28]

$$
p\left(\rho_{K}\right)= \begin{cases}\frac{\rho_{K}^{K-1} e^{-\left(\rho_{K} / \kappa\right)}}{\kappa^{K}(K-1) !}, & \text { for } \rho_{K} \geq 0 \\ 0 & \text { otherwise. }\end{cases}
$$

The cumulative distribution function $\Gamma(\cdot)$ can be given by

$$
\begin{aligned}
\Gamma\left(\rho_{K}\right) & =p\left(\rho_{K} \leq \mathrm{Th}\right)=\int_{0}^{\mathrm{Th}} p\left(\rho_{K}\right) d \rho_{K} \\
& =1-e^{-(\mathrm{Th} / \kappa)} \sum_{n=1}^{K} \frac{(\mathrm{Th} / \kappa)^{n-1}}{(n-1) !}
\end{aligned}
$$

where Th is the detection threshold.

2.3.2. Decision-Making Logic. In indoor environment, the signal is susceptible to multipath, blanking, and narrowband interference. A decision of all the interferences in spatial cooperative diversity technique depends on signal probability distribution model [11]. Related decision logic is a technique depicted in the literature review [29]. Effective decision logic and the selection of proper spatial cooperative diversity technique can enhance signal detection performance. In antenna diversity combination, $N$ input signals, after despreading denoted as $x_{i}$, received via $N$ diversity branches, are considered. In the detection process for GNSS signals, the performance can be evaluated through adoption of an individual binary hypothesis testing, defined as $H_{0}$ and $H_{1}$. $H_{0}$ corresponds to the scenario, when the incoming signal does not correspond to the hypothesis related to local replica $g_{i}$, whereas, under $H_{1}$ state, it is supposed that the frequency 
TABLE 1: Decision-making logic.

\begin{tabular}{lc}
\hline Combination mode & $K$ \\
\hline Mode 1: "SC" & $4<K \leq \operatorname{round}(N / 4), N>20$ \\
Mode 2: "SWC" & $4<K \leq \operatorname{round}(N / 2), 9 \leq N \leq 20$ \\
Mode 3: "MRC" & $2<K \leq 4, N<9$ \\
Mode 4: "EGC" & $K \leq 2, N<9$ \\
\hline
\end{tabular}

and code delay of the incoming signal are synchronized with the local replica. The detection structure for such binary hypothesis test results in the Likelihood Ratio Test (LRT), when the additive noise is zero mean Gaussian distribution [14]. The LRT for an antenna diversity structure consisting of $N$ branches can be considered as received signal estimator followed by a correlator. The different types of antenna combination methods can be performed under condition where correlation coefficient is equal to zero [30]. Assume the incoming signal includes desired signal component, and then the signal flag $D_{n}$ is set as one. Otherwise, the signal flag is set as zero. $K$ denotes the total times of $D_{n}=1(n=1,2, \ldots, N)$, which decides the type of antenna combination method. The definition of $K$ is the same as in (7). Table 1 demonstrates the decision-making logic process for the proposed scheme. The design criterion of decision-making logic depends on $K$ value that corresponds to the four operation modes including SC, SWC, MRC, and EGC.

2.3.3. Correlation Coefficient Evaluation. Each of the multiple antennas is separated spatially to achieve independent fading paths. The relative phase of the fading signal is obviously different at any two antennas because of the spatial separation, that is, antenna spacing $d$ between both the antennas. Thus, the correlation coefficient can be obtained through (2), which can be calculated by using the two signal correlations. Assume the received signal envelopes for the two antennas are $\alpha_{1}=\left|s_{01}\right|$ and $\alpha_{2}=\left|s_{02}\right|$, respectively, where $s_{01}$ and $s_{02}$ are the received complex signals on the first and second antenna, respectively, shown in (2). The correlation between the two antennas is given by [1]

$$
\begin{aligned}
\bar{\Lambda}_{\alpha_{1} \alpha_{2}}(\Delta \tau) & =E\left[\alpha_{1}\left(\tau^{1}\right) \alpha_{2}\left(\tau^{2}\right)\right] \\
& =E\left[\alpha_{1}\left(\tau^{1}\right) \alpha_{2}\left(\tau^{1}+\Delta \tau\right)\right] \\
& =\frac{\pi}{2}\left|\bar{\Lambda}_{z z}(0)\right|\left[1+\frac{1}{4} \frac{\left|\bar{\Lambda}_{z z}(\Delta \tau)\right|^{2}}{\left|\bar{\Lambda}_{z z}(0)\right|^{2}}\right],
\end{aligned}
$$

where $\Delta \tau=\left|\tau^{1}-\tau^{2}\right|$ describes time delay from antenna 1 to antenna $2 . \bar{\Lambda}_{z z}(0)$ represents the autocorrelation function of the received envelope $z=z_{I}+j z_{\mathrm{Q}}$. The detailed deduction of (16) is elaborated by Klukas et al. [1]. In (16), the $\left|\bar{\Lambda}_{z z}(\Delta \tau)\right|^{2}$ is given by

$$
\left|\bar{\Lambda}_{z z}(\Delta \tau)\right|^{2}=\bar{\Lambda}_{z_{I} z_{I}}^{2}(\Delta \tau)+\bar{\Lambda}_{z_{I} z_{Q}}^{2}(\Delta \tau)
$$

where $\bar{\Lambda}_{z_{I} z_{I}}^{2}(\Delta \tau)$ and $\bar{\Lambda}_{z_{I} z_{Q}}^{2}(\Delta \tau)$ are the real and imaginary parts of $\bar{\Lambda}_{z z}(\Delta \tau)$, respectively. Supposeing imaginary parts of correlation $\bar{\Lambda}_{z_{I} z_{Q}}^{2}(\Delta \tau)$ are equal to zero, then $\left|\bar{\Lambda}_{z z}(\Delta \tau)\right|^{2}=$ $\bar{\Lambda}_{z_{I} z_{I}}^{2}(\Delta \tau)$; meanwhile $\left|\bar{\Lambda}_{z z}(0)\right|^{2}=\bar{\Lambda}_{z_{I} z_{I}}^{2}(0)$, substituting above value into (16). Thus, the correlation $\bar{\Lambda}_{\alpha_{0} \alpha_{1}}(\Delta \tau)$ in (16) can be calculated as

$$
\bar{\Lambda}_{\alpha_{0} \alpha_{1}}(\Delta \tau)=\frac{\pi}{2}\left|\bar{\Lambda}_{z_{I} z_{I}}(0)\right|\left[1+\frac{1}{4} \frac{\bar{\Lambda}_{z_{I} z_{I}}^{2}(\Delta \tau)}{\bar{\Lambda}_{z_{I} z_{I}}^{2}(0)}\right] .
$$

Utilizing the Bessel function expression, the $\bar{\Lambda}_{z z}(\Delta \tau)$ can be expressed as

$$
\left|\bar{\Lambda}_{z z}(\Delta \tau)\right|^{2}=\bar{\Lambda}_{z_{I} z_{I}}^{2}(\Delta \tau)=\frac{\bar{P}^{2}}{4} J_{0}^{2}\left(2 \pi \frac{d}{\lambda}\right),
$$

where $d$ denotes the spacing between first antenna and second antenna, $\lambda$ is the wavelength of the incoming plane wave, and $J_{0}(\cdot)$ represents zero-order Bessel function of first kind $[31,32] . \bar{P}$ indicates the total received envelope power. Using (19), the autocovariance function is given by

$$
\begin{aligned}
\Re(d) & =E\left[\alpha_{0}\left(\tau^{0}\right) \alpha_{1}\left(\tau^{1}\right)\right]-E\left[\alpha_{0}\left(\tau^{0}\right)\right] E\left[\alpha_{1}\left(\tau^{1}\right)\right] \\
& =\frac{\pi}{2}\left|\bar{\Lambda}_{z z}(0)\right|\left[1+\frac{1}{4} \frac{\bar{\Lambda}_{z z}^{2}(\Delta \tau)}{\bar{\Lambda}_{z z}^{2}(0)}\right]-\frac{\pi}{2} \bar{\Lambda}_{z z}^{2}(0) \\
& =\frac{\pi}{8} \frac{\bar{\Lambda}_{z z}^{2}(\Delta \tau)}{\bar{\Lambda}_{z z}(0)}=\frac{\pi \bar{P}}{16} J_{0}^{2}\left(2 \pi \frac{d}{\lambda}\right) .
\end{aligned}
$$

After normalization, the antenna correlation between the first and second antenna elements is expressed as

$$
\overline{\mathfrak{R}}_{12}(d)=J_{0}^{2}\left(2 \pi \frac{d}{\lambda}\right) .
$$

The envelope correlation between the two receiver channels is calculated and compared with theoretical values found by Stüber [31]. The resulting output is coherently in-phase $I_{n}$ and quadrature $Q_{n}$ integrated prior to temporal or spatial processing after code and carrier removal. Assume a coherent integration output of $T_{c}$ seconds for the first and second antennas is $x_{0}[k]=\sum_{M} I_{0}+j Q_{0}$ and $x_{1}[k]=\sum_{M} I_{1}+j Q_{1}$ respectively; in that case their envelopes are $x_{0}=\left|x_{0}[k]\right|$ and $x_{1}=\left|x_{1}[k]\right|$, respectively; then the envelope correlation between the two antennas is depicted in terms of meanremoved versions of $x_{0}$ and $x_{1}$ as

$$
\begin{aligned}
\overline{\mathfrak{R}}_{12}^{\text {True }}(d) & =\frac{E\left[\left(x_{0}-\bar{m}_{y_{0}}\right)\left(x_{1}-\bar{m}_{x_{1}}\right)\right]}{\sqrt{\operatorname{var}\left(x_{0}-\bar{m}_{x_{0}}\right)} \cdot \sqrt{\operatorname{var}\left(x_{1}-\bar{m}_{x_{1}}\right)}} \\
& =\frac{E\left[\left(x_{0}-\bar{m}_{y_{0}}\right)\left(x_{1}-\bar{m}_{y_{1}}\right)\right]}{\sqrt{E\left[\left(x_{0}-\bar{m}_{x_{0}}\right)^{2}\right]} \cdot \sqrt{E\left[\left(x_{1}-\bar{m}_{x_{1}}\right)^{2}\right]}} .
\end{aligned}
$$

The depiction in (22) holds true for a uniform angle of arrival distribution in azimuth and omnidirectional receiving 
antennas matched to the polarization of the incoming radio wave. The symbol $\operatorname{var}(\cdot)$ depicts the variance calculation operator with deviation $\bar{m}_{(\cdot)}$. The envelope correlation of signals in (22) received by any two antennas suffers from several factors such as the mutual coupling, radiation pattern, direction of arrival of the radio frequency (RF) wave, and antenna gain.

\section{Performance Criteria}

The proposed scheme combines the polarization diversity and pattern diversity to form the desired array gain pattern. Because the right-hand circular polarization (RHCP) or linear polarization is used in common GNSS antenna, the antenna spacing is only an arrangement factor used to avoid the effect of mutual coupling and cross-polarization [14]. Therefore, this section illustrates the performance evaluation criteria of the proposed system.

3.1. Signal Detection Probability. As detection performance of the spatial combination technique is a function of only the probability of false alarm $P_{\mathrm{fa}}$ and the probability of detection $P_{d}$, thus, assume that, under $H_{1}$ hypothesis, the frequency and code phase of the incident signal are synchronized with locally generated signal. Otherwise, the signal is not presented (under $H_{0}$ hypothesis). For single antenna case, the detection statistic is a chi-square two-degrees-of-freedom central probability density function random variable for both $H_{1}$ and $H_{0}$ conditions. As a result, the false alarm probability $P_{\mathrm{fa}}$ and detection probability $P_{d}$ are shown as follows [33]:

$$
\begin{gathered}
P_{\mathrm{fa}}(\mathrm{Th})=\bar{\Gamma}\left(T_{y}>\mathrm{Th} \mid H_{0}\right)=\exp \left(-\frac{\mathrm{Th}}{2 \sigma^{2}}\right), \\
P_{d}(\mathrm{Th})=\bar{\Gamma}\left(T_{y}>\mathrm{Th} \mid H_{1}\right)=\exp \left(-\frac{\mathrm{Th}}{2 \sigma^{2}(\mathrm{SNR}+1)}\right),
\end{gathered}
$$

where $\bar{\Gamma}(\cdot)$ is probability function. As detection performance of a multiple antenna system, the EGC is explained with uncorrelated channel condition: that is, $\Psi_{m n}^{2}=0$,

$$
\begin{gathered}
P_{\mathrm{fa}}(\mathrm{Th})=\left(1+\frac{\mathrm{Th}}{2}\right) \exp \left(-\frac{\mathrm{Th}}{2}\right), \\
P_{d}(\mathrm{Th})=\bar{\Gamma}\left(T_{y}>\mathrm{Th} \mid H_{1}\right) \\
=\left(1+\frac{\mathrm{Th}}{2(\mathrm{SNR}+1)}\right) \exp \left(-\frac{\mathrm{Th}}{2(\mathrm{SNR}+1)}\right) .
\end{gathered}
$$

Figure 4 demonstrates the distribution of signal detection probability using different switch methods under $P_{\mathrm{fa}}=1 \%$. The diagram shows that the signal detection probability of proposed scheme can be regarded as upper and lower boundary range. That is because the proposed decision logic is based on Table 1. Thus, different combinations of the antennas are selected and adopted.

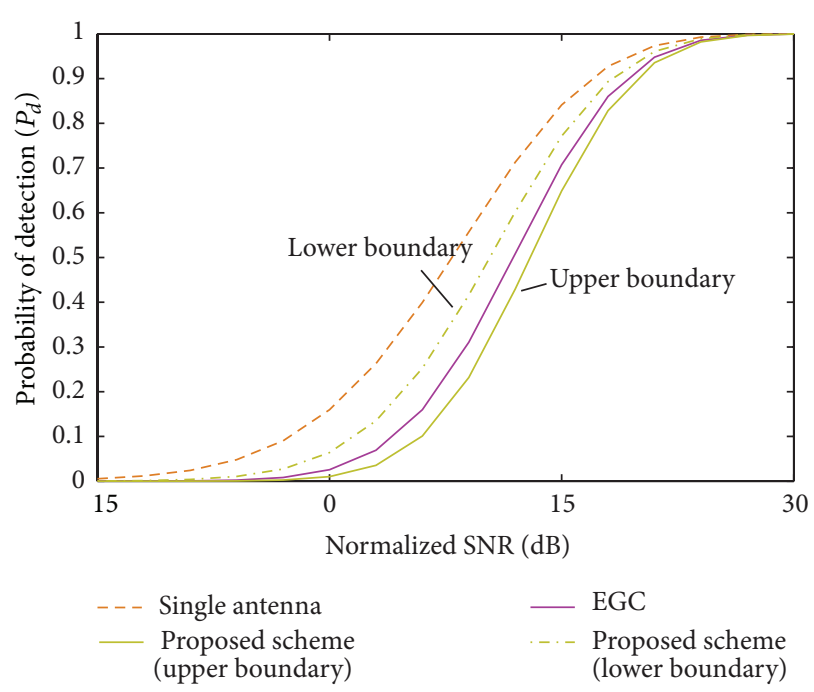

FIGURE 4: The probability of detection for different spatial combination method.

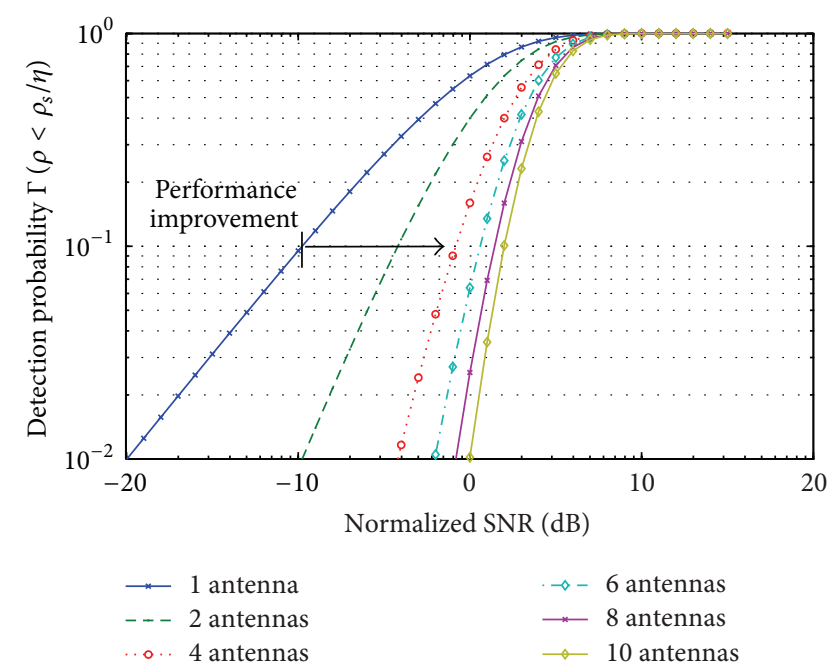

FIGURE 5: Probability of Rayleigh fading signals for different numbers of diversity antennas.

3.1.1. Diversity Gain. The performance analysis of spatial cooperative diversity technique can be assessed through calculated diversity gain. The evaluation standard lies in the difference between the acquired SNR of single antenna system and that of the spatial cooperative diversity technique. For example, in multipath environment, the channel is Rayleigh. Then, the cumulative distribution function (CDF) can be calculated as suggested earlier [34]:

$$
\Gamma\left(\rho<\frac{\rho_{s}}{\eta}\right)=1-e^{\left(\rho_{s} / \eta\right)},
$$

where $\rho_{s} / \eta$ is the normalized SNR or threshold, $\eta$ is the average of SNR, $\rho$ depicts the calculated instantaneous SNR, and $\Gamma\left(\rho<\rho_{s} / \eta\right)$ represents the probability of SNR below threshold $\rho_{s} / \eta$. The choice of $K$ antenna indicates that the signal detection probability of whole antenna system 


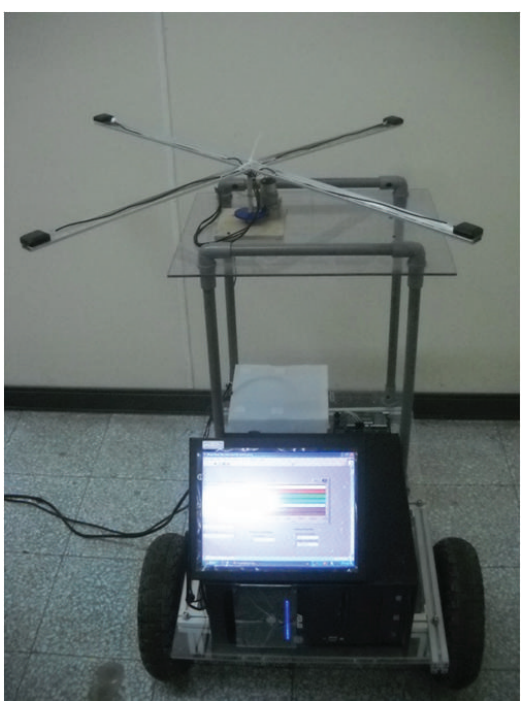

(a)

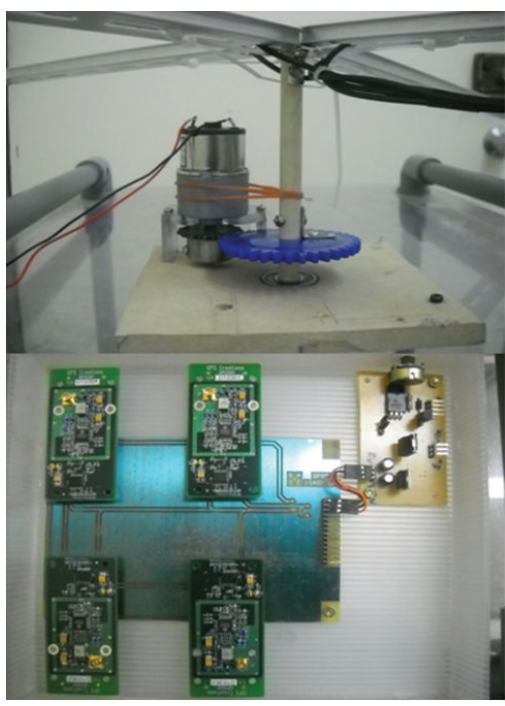

(b)

Figure 6: Platform of the moving vehicle.

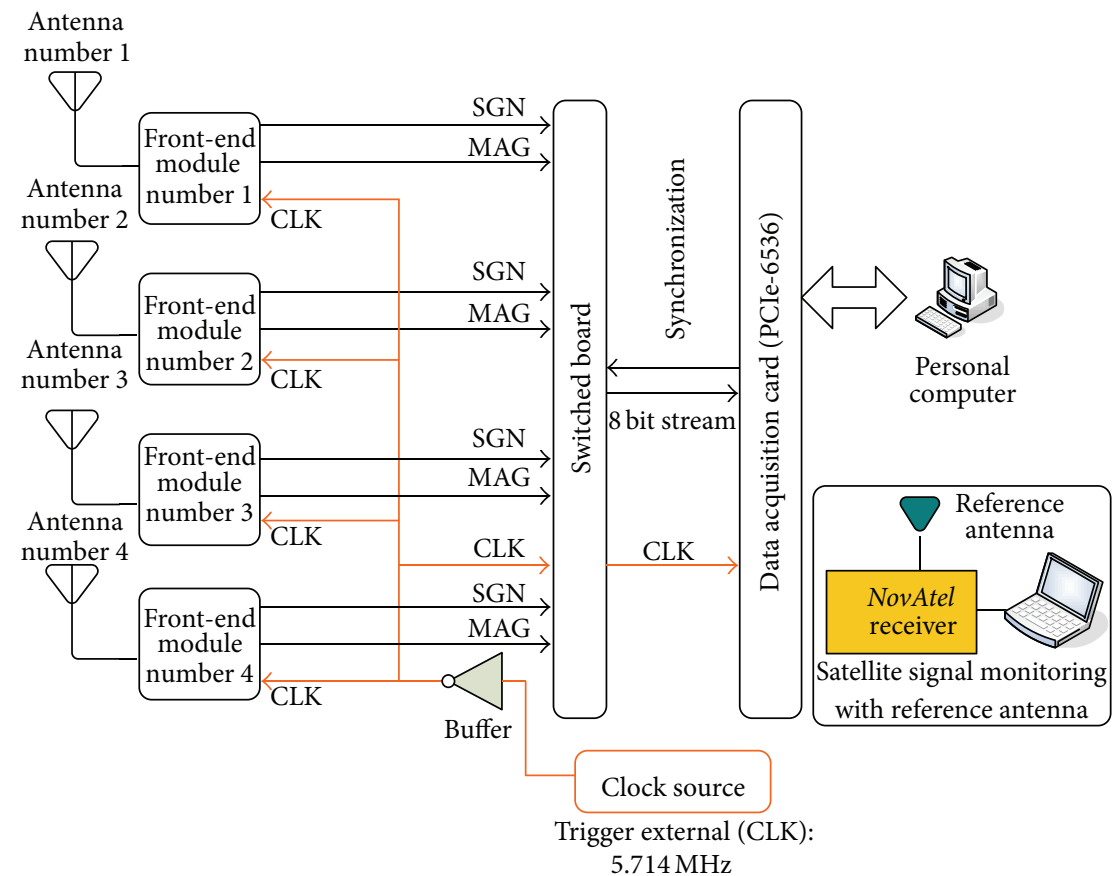

FIGURE 7: Schematic of the experiment setup.

is $K$ times. Assuming that the $K$ antenna branches have independent signals and equal mean SNRs, the probability of all branches having a SNR below $\rho_{s}$ equals the probability for a single antenna branch increased to the power $K$ as

$$
\Gamma\left(\rho<\frac{\rho_{s}}{\eta}\right)_{K}=\left(1-e^{\rho_{s} / \eta}\right)^{K} .
$$

Usually $\Gamma\left(\rho<\rho_{s} / \eta\right)$ is set as $1 \%$. At low instantaneous SNR $(\rho \ll \eta),(26)$ approximates $\left(e^{\rho_{s} / \eta}\right)^{K}$ when $\Gamma\left(\rho<\rho_{s} / \eta\right)$ is given; diversity gain can be calculated as

$$
\bar{\rho}_{D}=\rho_{g}-\rho_{1},
$$

where $\rho_{g}$ represents the SNR using cooperative diversity technique, whereas $\rho_{1}$ stands for the SNR of the first antenna without using cooperative diversity technique. The result of 


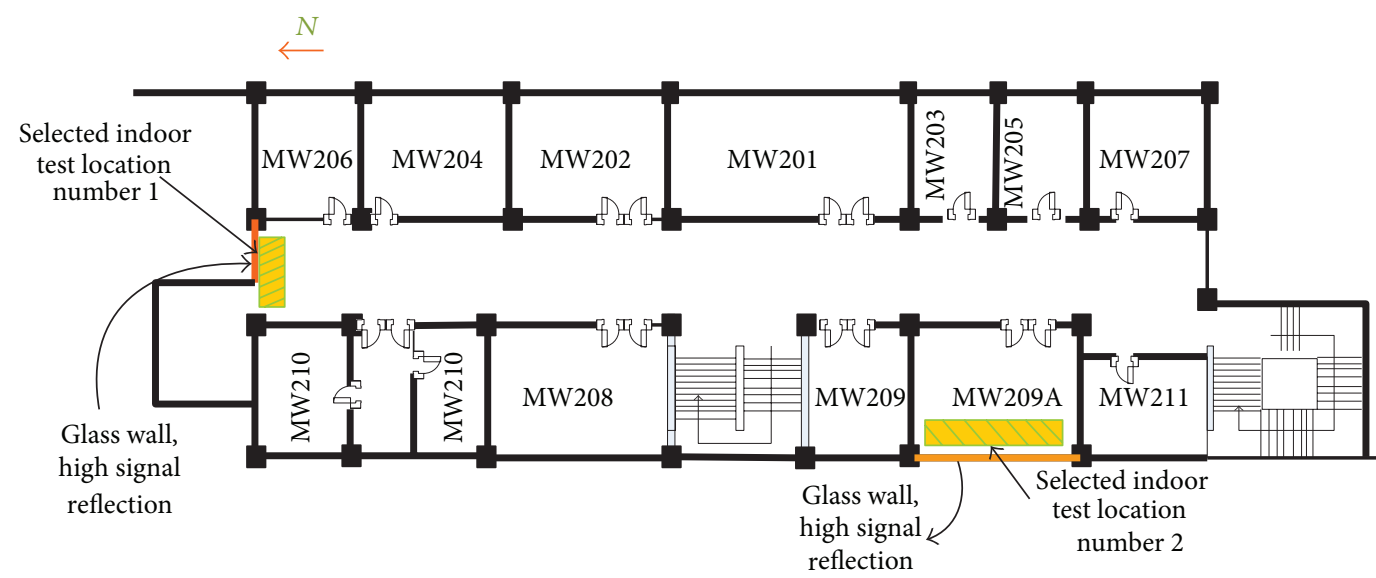

FIgURE 8: Experimental test location in BIOME building, NPUST.

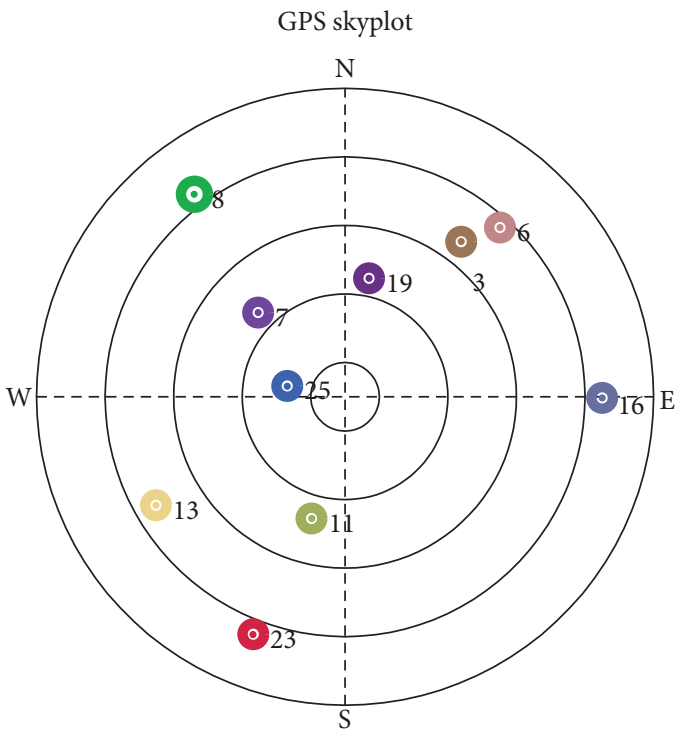

FIGURE 9: Sky-view plot of GNSS.

diversity gain can evaluate the signal detection performance of the whole system. In GNSS application, the diversity gain measurements initially involve the estimation of SNR at the output of the coherent integration. Thus, here the post-SNR for the single and multiple antenna channels combination method is regarded as the deflection coefficient. Thus, (27) is rewritten as follows [33]:

$$
\rho_{D}^{2}=\frac{2\left[E\left[T_{x} \mid H_{1}\right]-E\left[T_{x} \mid H_{0}\right)\right]^{2}}{\operatorname{var}\left(T_{x} \mid H_{0}\right)+\operatorname{var}\left(T_{x} \mid H_{1}\right)},
$$

where $T_{x}$ represents the test statistic. Because the detection statistics are no longer modeled as Gaussian random variables, the deflection coefficient offers only a rough measure of diversity gain.

Figure 5 shows the reduction of the probability of fading below a given threshold or normalized SNR when increasing the number of antennas. This figure also shows that, for

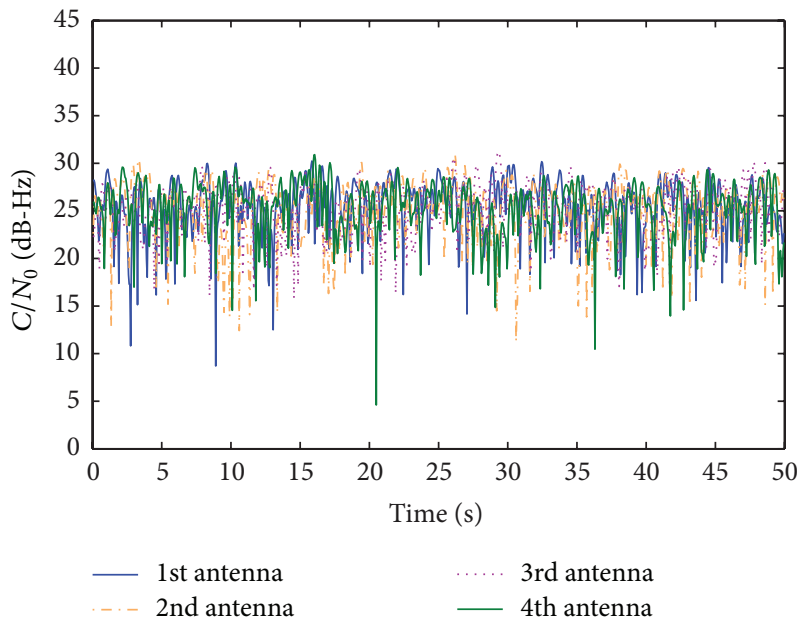

FIgURE 10: $C / N_{0}$ plots for the two antennas at the indoor fading environment.

$\Gamma\left(\rho<\rho_{s} / \eta\right)=10 \%$ reliability level, there are diversity gains of $9.2 \mathrm{~dB}$ and $7.3 \mathrm{~dB}$ for the two-antenna and four-antenna selection combiners, respectively.

3.1.2. Correlation Coefficient. Furthermore, the spatial relationship between any two antennas is used to calculate the spatial correlation. Ideally, the spatial correlation function between any two antennas is low, and the difference of calculated SNR among each antenna model is small. The square value of complex correlation coefficient is quantified in the following equation:

$$
\kappa^{2}=\frac{E\left[\Lambda_{1} \Lambda_{2}^{*}\right]^{2}}{E\left[\Lambda_{1} \Lambda_{1}^{*}\right] E\left[\Lambda_{2} \Lambda_{2}^{*}\right]},
$$

where $\Lambda_{1}$ and $\Lambda_{2}$ are zero mean complex values of the correlator output of each received signal channel. "*" is the complex conjugate operator. Higher correlation coefficient in spatial diversity may result in lower diversity gain. 


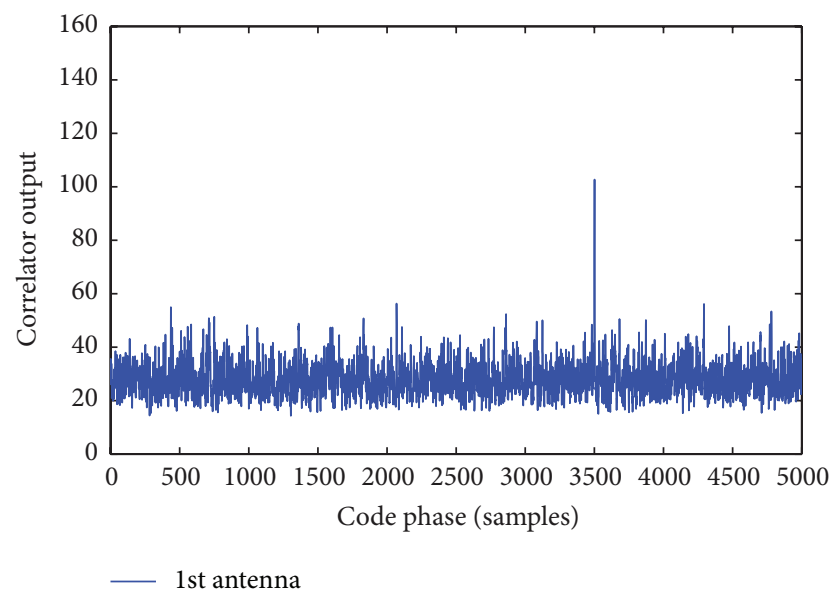

(a)

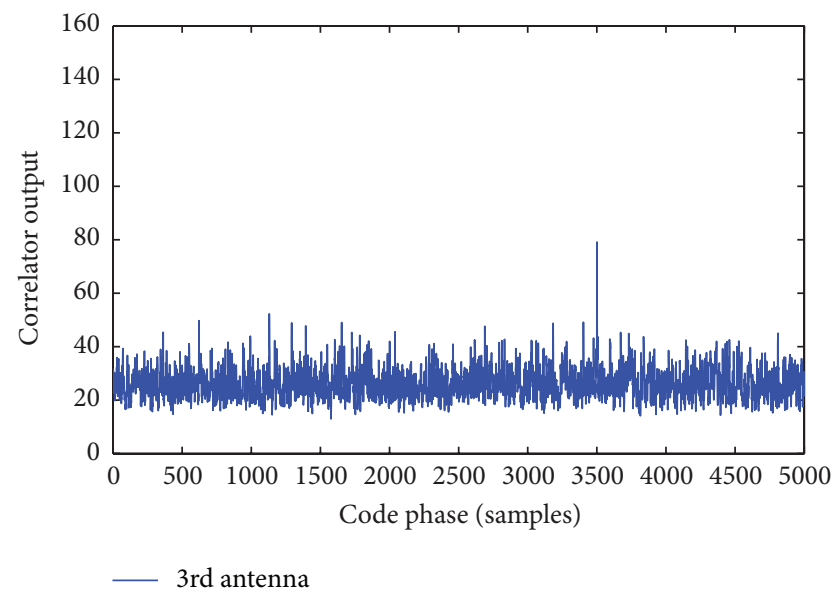

(c)

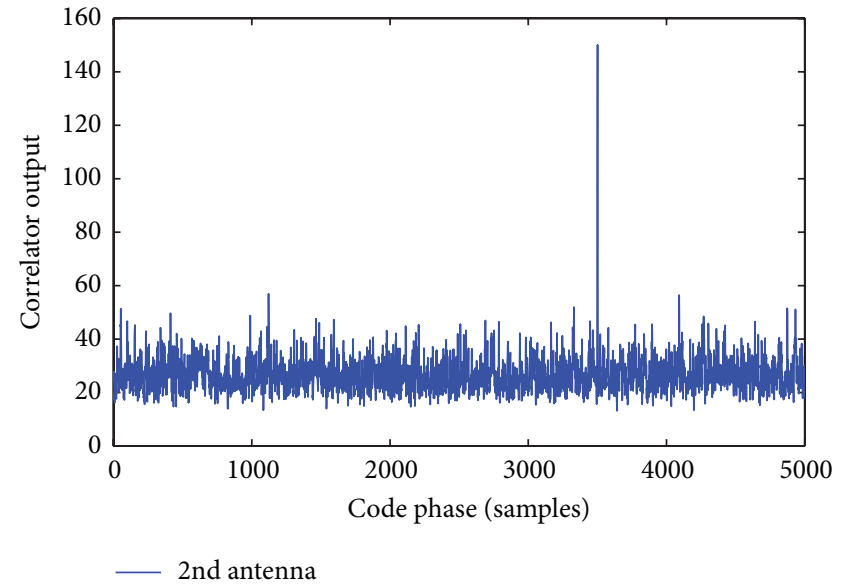

(b)

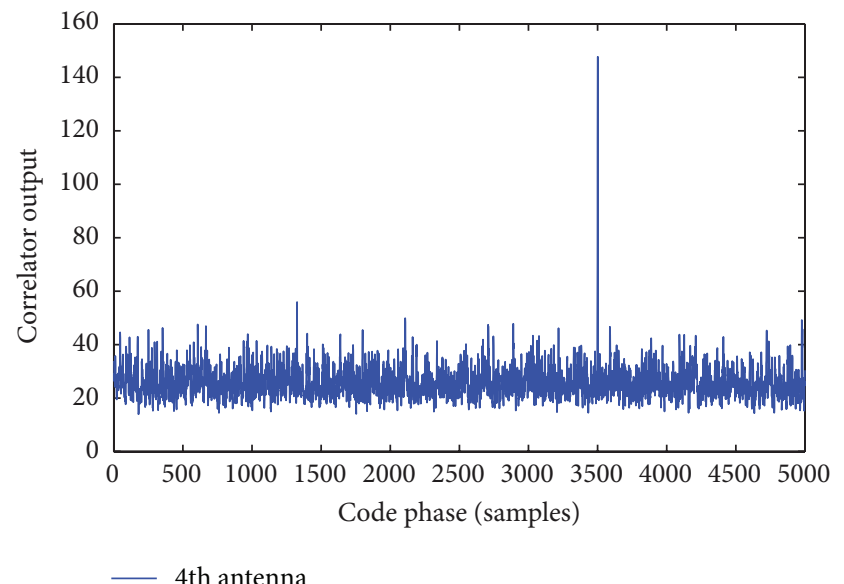

(d)

FIGURE 11: Weak GNSS signal acquisition results from each antenna branch.

\section{Test Hardware}

This chapter describes hardware setup that is the use of multiple antennas for different spatial combing including switching, selection, equal gain, and maximum gain method. The establishment of antenna array system is also demonstrated in this chapter.

4.1. Hardware Specification. Figure 6 shows the mobile robot appearance. The robot is equipped with motor module, and the use of motor and gear is adopted to control the rotation speed of the antenna array. The RF front-end module and NI CB-2162 are mounted on computer side, and ends of the antennas are connected to the GPS501 hardware box. Four commercial GPS front-ends are adopted to filter, downconvert, and digitize incoming RF signal to obtain digital samples. A master clock is utilized to control the synchronization among four different channels so that the samples can be acquired simultaneously. The digitized samples are acquired at the rate of $5.714 \mathrm{MHz}$. The digital samples are obtained using a PCI-based data acquisition board (PCI6536) through a data acquisition software (NI LabView). The data samples from each antenna element are stored and postprocessed for interference mitigation analysis. Figure 7 shows the structure of array system hardware. Firstly, switched board (NI CB-2162) acquires the "SGN" and "MAG" binary digital signal from the four RF front-end modules. After that, the 8 bit signal, which consists of four groups of "SGN" and "MAG" signal, is sent to an NI PCIe-6536 high-speed digital I/O cards and then stored in the personal computer. The NovAtel reference antenna is adopted to estimate the Doppler frequency, code phase, and navigation data bits, and these estimates are utilized to demodulate the GPS digital intermediate frequency (IF) data with respect to the indoor antenna.

4.2. Experimental Setup. The two selected indoor locations are situated at biomechatronics engineering building, namely, the BIOME building of Pingtung University of Science and Technology (NPUST). Figure 8 illustrates the schematic plan view of the second floor of the building and the indoor test locations. The first location is situated in a corridor, and the other location is inside a room of the 2 nd floor. The indoor test location number 1 inside the corridor has its southern 
view blocked with the building wall, but northern of the antenna is open through the glass windows. For verification purposes, a second location on the same floor of the same building is also investigated. The patch antenna indoors are facing the glass window panes towards the western direction. Figure 9 depicts the sky-view plot at the time as recorded by the reference receiver (NovAtel Inc.) located at the rooftop. The roof reference antenna is stationary; the indoor patch antennas are mounted on a rotation platform. The antenna spacing is set as $1 \lambda$. The experiments are divided into static and dynamic scenarios, respectively. After that, the received data is stored and processed using MATLAB software for signal acquisition and tracking.

\section{Experimental Results}

This chapter analyzes the performance of proposed scheme through experiments. The initial step is to have a mobile robot to move to the designated position. Upon arrival, the mobile robot stops moving and starts its experiment of static signal reception. The duration of static data is half an hour. The dynamic rotation speeds are 1, 4, and $20 \mathrm{rpm}$. The reception time in terms of dynamic data is half an hour. The above data are utilized for the performance analysis using the following three steps. First of all, the indoor fading scenario for all of the antennas is demonstrated, where the $C / N_{0}$ distribution characteristics indicate the indoor fading scenario with respect to envelope correlation and Rician factor. The envelope correlation is compared with the theoretical correlation coefficient as the antenna spacing varies. Secondly, the effects of cooperative combination are depicted. The effects of EGC and the proposed scheme are particularly compared. Besides, the comparison with single antenna case is also conducted, and post-SNR improvements for the proposed decision scheme are also described. At last, analyses with receiver operating characteristics (ROC) curves with regard to improvement in detection probability in contrast to that of the single antenna processing are demonstrated.

5.1. Fading Environment. It is necessary to extend coherent integration time to obtain high-sensitivity operation due to weak and severe nonline of sight (NLOS) signal conditions in indoors. Coherent and noncoherent integration times are $8 \mathrm{~ms}$ and $1 \mathrm{sec}$, respectively. As an example of indoor fading characteristics, $C / N_{0}$ over the time for PRN 3 corresponding to the dataset with $1 \lambda$ antenna spacing is shown in Figure 10. The average $C / N_{0}$ for all of the antennas is roughly $25.1 \mathrm{~dB}$ Hz. Figure 11 demonstrates the acquisition result of a weak GPS signal (PRN 3) from each antenna with $1 \lambda$ antenna spacing, where the correlation peak is also zoomed in plots of Doppler frequency and code offset.

In Figure 12, the average antenna correlation acquired through experimental measurements supports the theoretical envelope correlation prediction demonstrated in (12). This figure illustrates that the measured antenna envelope correlation is lower than the theoretical correlation curve, especially in terms of smaller antenna spacing. This is because the indoor patch antenna employed in this paper is not an ideal

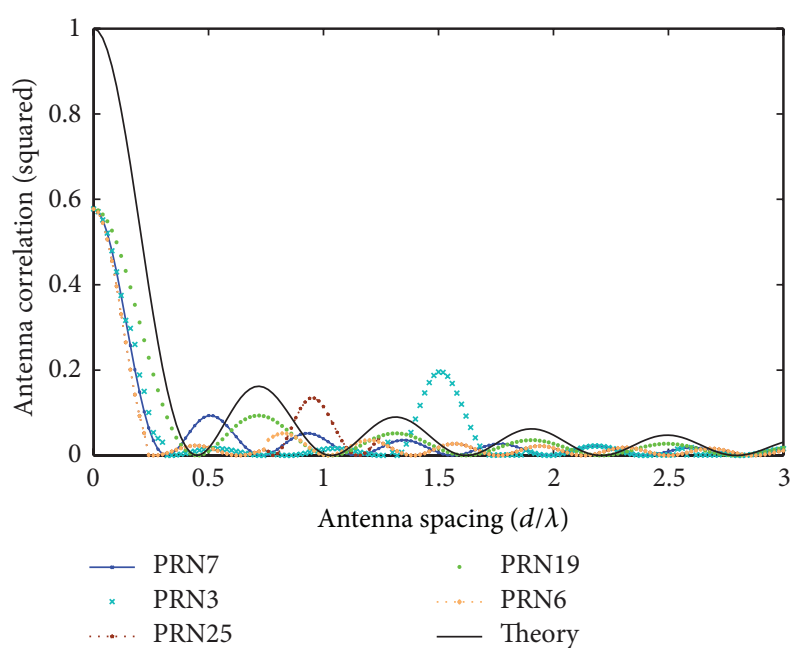

FIGURE 12: Squared envelope correlation as a function of antenna spacing, indoor location.

one, and the radiation pattern of the indoor patch antenna is not omnidirectional. Figure 12 depicts that the measured squared magnitude envelopes maintain a similar shape for all of the available pseudorandom noise (PRNs), but they differ with respect to the actual correlation value. This is because the actual antenna envelope correlation counts on the angular multipath spread in addition to the antenna characteristics.

Figure 13 depicts the deflection coefficient measures for the single and proposed decision schemes corresponding to the indoor data set of $0.5 \lambda$ antenna spacing for PRN 19. The cumulative distribution function is also plotted for the same dataset. The deflection coefficient for the spatial combination techniques has gone through a lower number of fades in contrast to the single antenna processing. Correspondingly, the deflection coefficient values increase drastically for a smaller value of cumulative probability. The performance gap between single antenna and diversity antennas and the performance improvement results from the diversity gain with cooperative signal combination are also shown in Figure 13.

Figure 14 shows the results of a research on the achievable post-SNR for the different antenna spacings averaged over all PRNs. A coherent integration period of $5 \mathrm{~ms}$ and a total observation period of $1 \mathrm{~s}$ for noncoherent summation are taken into account. Both EGC and proposed scheme show better performance than single antenna performance. Nevertheless, proposed scheme presents a slightly superior performance in contrast to that of EGC. This is because the performance of proposed scheme is affected by the correlation coefficients between the antennas. The correlation coefficient is reduced by increasing the antenna spacing, and both EGC and proposed scheme present similar performance.

5.2. Post-SNR. Table 2 illustrates the average post-SNR results that use different types of cooperative diversity method under the worst case. In Table 2, the proposed 


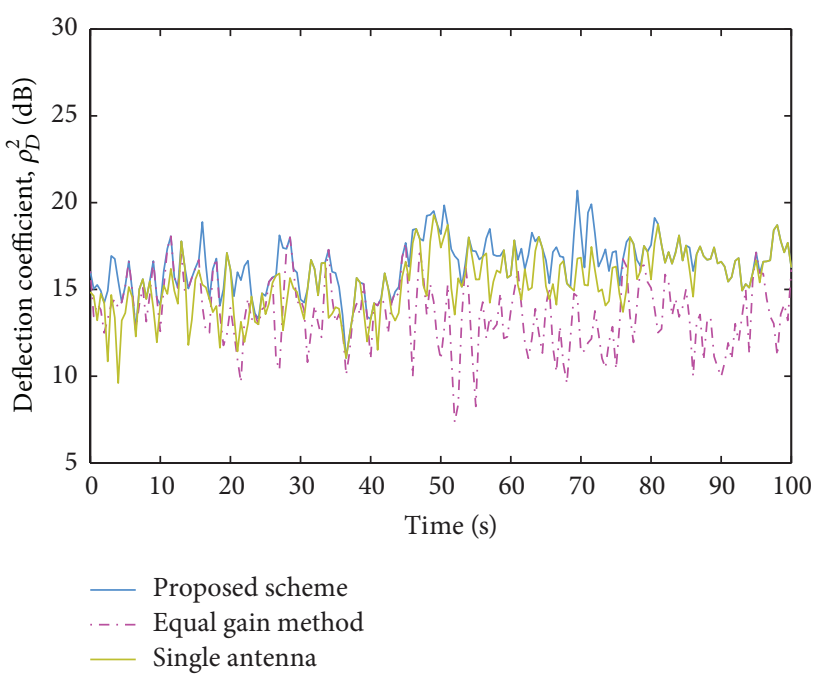

(a)

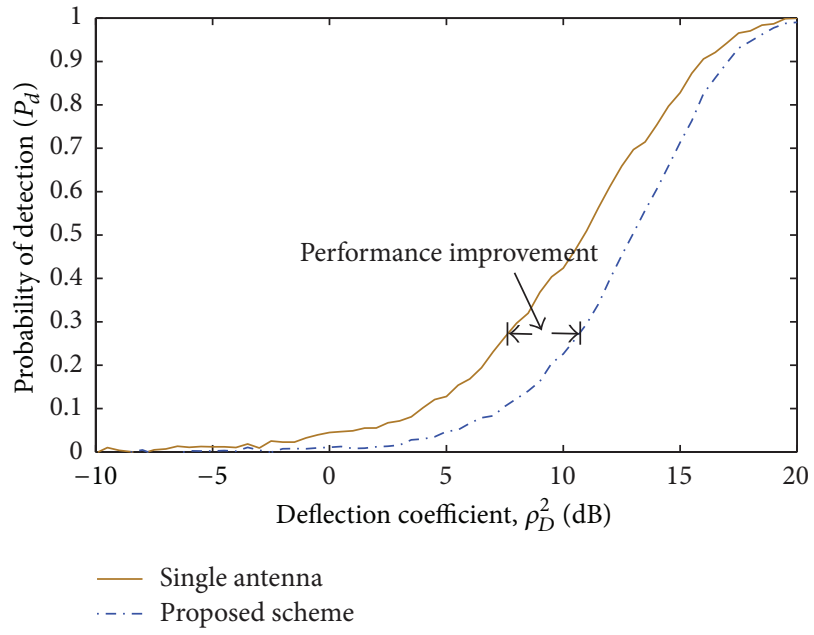

(b)

Figure 13: (a) Deflection coefficient as a function of time. (b) Probability of detection versus deflection coefficient, PRN 19, $d=0.5 \lambda$.

TABLE 2: Results of static and dynamic experiment in worst case $\left(T_{c}=5 \mathrm{~ms}\right.$, noncoherent integration time is $\left.0.5 \mathrm{sec}\right)$.

\begin{tabular}{|c|c|c|c|c|c|c|c|c|}
\hline \multirow[b]{2}{*}{$\begin{array}{l}\text { Average post-SNR } \\
\text { (Th: } 17 \mathrm{~dB})\end{array}$} & \multicolumn{4}{|c|}{ Single antenna case } & \multirow[b]{2}{*}{ SWC } & \multirow[b]{2}{*}{ MRC } & \multirow[b]{2}{*}{ EGC } & \multirow[b]{2}{*}{ Proposed scheme } \\
\hline & $\begin{array}{l}\text { 1st antenna } \\
\text { (North) }\end{array}$ & $\begin{array}{l}\text { 2nd antenna } \\
\text { (East) }\end{array}$ & $\begin{array}{l}\text { 3rd antenna } \\
\text { (South) }\end{array}$ & $\begin{array}{l}\text { 4th antenna } \\
\text { (West) }\end{array}$ & & & & \\
\hline \multicolumn{9}{|c|}{ Static experiment (PRN 19) } \\
\hline $0.5 \lambda$ & $\times$ & 17.3 & 17.3 & 17.7 & $17.3 \sim 17.7$ & 19.5 & 18.3 & 19.5 \\
\hline $1 \lambda$ & 17.9 & 17.8 & 17.9 & 18.3 & $17.8 \sim 18.3$ & 20.9 & 18.6 & 20.9 \\
\hline $2.5 \lambda$ & $\times$ & $\times$ & $\times$ & $\times$ & $\times$ & 19.1 & 18.0 & 19.1 \\
\hline \multicolumn{9}{|c|}{ Dynamic experiment (PRN 19; antenna spacing: $1 \lambda)$} \\
\hline $1 \mathrm{rpm}$ & 17.7 & 17.5 & 17.7 & 17.9 & $17.5 \sim 17.9$ & 18.8 & 18.5 & 18.8 \\
\hline $4 \mathrm{rpm}$ & 17.7 & 17.3 & 17.1 & 17.0 & $17.1 \sim 17.7$ & 17.5 & $\times$ & 17.5 \\
\hline $20 \mathrm{rpm}$ & $\times$ & $\times$ & 17.4 & 17.1 & $\times$ & 18.7 & $\times$ & 18.7 \\
\hline
\end{tabular}

“X”: unlocked.

scheme with $1 \lambda$ antenna spacing provides $2.3 \mathrm{~dB}$ post-SNR performance enhancement in contrast to EGC method under the static experiment (without antenna array rotation). The performance of proposed scheme is the same as that of MRC method. In addition, only the use of proposed scheme with $1 \lambda$ antenna spacing and $20 \mathrm{rpm}$ can acquire the desired signal under the dynamic experiment (with antenna array rotation).

5.3. Detection Performance. Performance evaluation with respect to the deflection coefficient is only a rough account of the improvements, not an accurate measure. Thus, the detection performance has been conducted based on the indoor test data in the form of ROC. The coherent integration period of $100 \mathrm{~ms}$ results in good probability of the detection. So as to acquire test statistics for $H_{1}$, that is, under the presence of a signal, the correct code phase estimate is adopted and the corresponding detection output is computed. Incorrect code phase is employed to obtain the test statistics under $H_{0}$ condition. The average post-SNR values for the PRN processed in the dataset for the static experiment are in the range from 17 to $20 \mathrm{~dB}-\mathrm{Hz}$. Figure 15 depicts the CDF of the detection output for single antenna and cooperative antennas at $1 \lambda$ antenna spacing. The detection output was based on $100 \mathrm{~ms}$ coherent integration time and $6 \mathrm{sec}$ noncoherent integration time. Under $H_{0}$ and $H_{1}$ hypotheses, single antenna performance and cooperative combination with EG and proposed scheme are demonstrated. Figure 15 readily presents the advantages of adopting multiple antennas for signal detection purposes. For any given percentage of time, the proposed scheme presents slightly superior detection performance than that of EGC.

\section{Conclusion}

This research demonstrates the dynamic cooperative scheme to enhance the signal detection performance with regard to multiple antennas under indoor environment. Simulation result illustrates that the use of multiple antennas with decision logic scheme can effectively improve post-SNR performance in contrast to single antenna case. Meanwhile, 


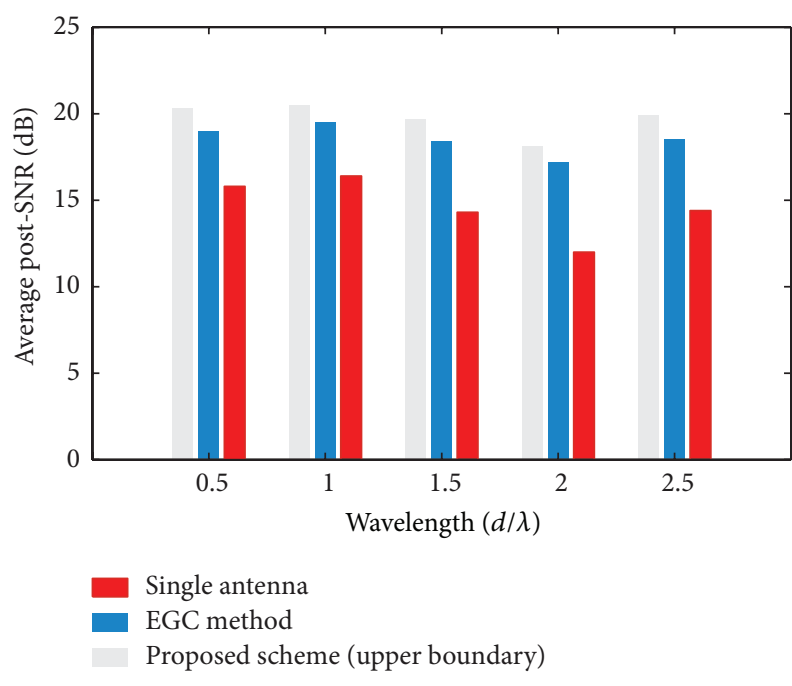

FIgURE 14: Averaged post-SNR as a function of antenna spacing (indoor location; $T_{c}=5 \mathrm{~ms}$, noncoherent integration time is $1 \mathrm{sec}$, PRN 19).

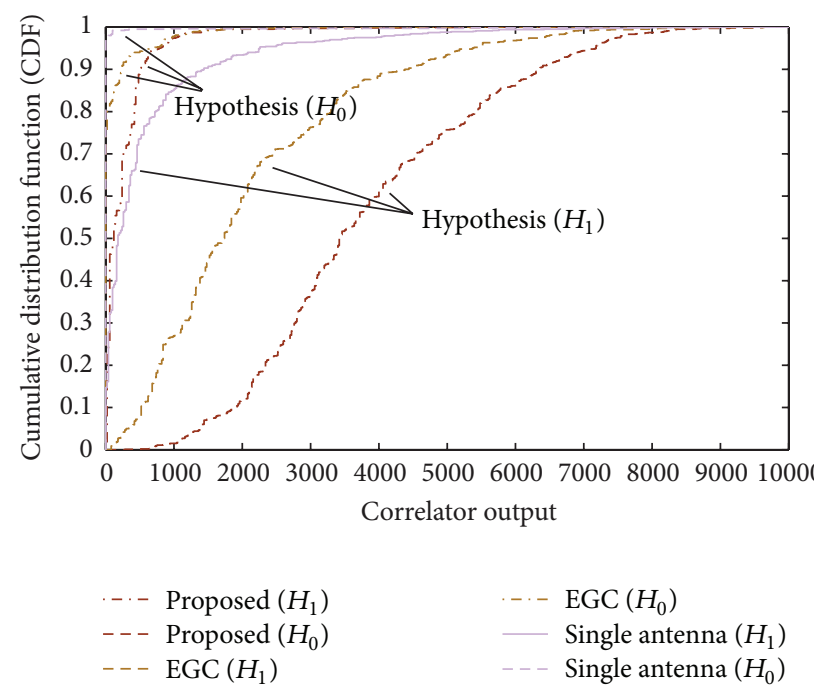

FIGURE 15: CDF of detection output in the case of four antennas, $T_{c}=100 \mathrm{~ms}$, and noncoherent integration time is $6 \mathrm{sec}$, in the indoor location.

real-time data reception is conducted under indoor environment. It is successfully verified that the proposed scheme indeed effectively enhances signal detection probability and improve post-SNR. In the future, this technique can combine beamforming algorithm to be applied to the multinavigation satellite signal reception and the interference mitigation system.

\section{Acknowledgments}

Many thanks are due to the editor and reviewers for their valuable comments to refine this paper. Besides, the authors would like to thank the National Science Council in Taiwan for supporting this research under Grant NSC 102-2221-E020-007.

\section{References}

[1] R. Klukas, O. Julien, L. Dong, M. E. Cannon, and G. Lachapelle, "Effects of building materials of UHF ranging signals," GPS Solutions, vol. 8, no. 1, pp. 1-18, 2004.

[2] B. Peterson, D. Bruckner, and S. Heye, "Measuring GPS signals indoors," in Proceedings of the 10th International Technical Meeting of the Satellite Division of the Institute of Navigation (ION GPS '97), pp. 615-624, September 1997.

[3] F. van Digglen and C. Abraham, "Indoor GPS technology," in Proceedings of CTIA Wireless-Agenda, pp. 1-10, 2001.

[4] F. van Diggelen, "Indoor GPS theory \& implementation," in Proceedings of the IEEE Position Location and Navigation Symposium, pp. 240-247, April 2002.

[5] J.-A. Tsai, R. M. Buehrer, and B. D. Woerner, "Spatial fading correlation function of circular antenna arrays with Laplacian energy distribution," IEEE Communications Letters, vol. 6, no. 5, pp. 178-180, 2002.

[6] G. J. Foschini, "Layered space-time architecture for wireless communication in a fading environment when using multielement antennas," Bell Labs Technical Journal, vol. 1, no. 2, pp. 41-59, 1996.

[7] H. Bolcskei, D. Gesbert, C. B. Papadias, and A. van der Veen, Space-Time Wireless Systems-From Array Processing to MIMO Communications, Cambridge University Press, Cambridge, UK, 2006.

[8] R. L. Fante and J. J. Vaccaro, "Wideband cancellation of interference in a GPS receive array," IEEE Transactions on Aerospace and Electronic Systems, vol. 36, no. 2, pp. 549-564, 2000.

[9] S.-J. Kim and R. A. Iltis, "STAP for GPS receiver synchronization," IEEE Transactions on Aerospace and Electronic Systems, vol. 40, no. 1, pp. 132-144, 2004.

[10] C.-L. Chang, "Self-tuning synthesis filter against mutual coupling and interferences for GNSS and its implementation on embedded board," EURASIP Journal on Advances in Signal Processing, vol. 2010, Article ID 123625, 13 pages, 2010.

[11] C. L. Chang, "Multiplexing scheme for anti-jamming global navigation satellite system receivers," IET Radar, Sonar \& Navigation, vol. 6, no. 6, pp. 443-457, 2012.

[12] C. B. Dietrich Jr., K. Dietze, J. R. Nealy, and W. L. Stutzman, "Spatial, polarization, and pattern diversity for wireless handheld terminals," IEEE Transactions on Antennas and Propagation, vol. 49, no. 9, pp. 1271-1281, 2001.

[13] C. C. Chiau, Study of the diversity antenna array for the MIMO wireless communication systems [Ph.D. thesis], Queen Mary University of London, London, UK, 2006.

[14] Y. Gao, C. C. Chiau, X. Chen, and C. G. Parini, "Design of diversity antenna array for Galileo/GPS receivers," in Proceedings of the European Conference on Antennas and Propagation (EuCap '06), pp. 1-5, Nice, France, November 2006.

[15] A. Broumandan, J. Nielsen, and G. Lachapelle, "Indoor GNSS signal acquisition performance using a synthetic antenna array," IEEE Transactions on Aerospace and Electronic Systems, vol. 47, no. 2, pp. 1337-1350, 2011.

[16] M. Zaheri, A. Broumandan, V. Dehghanian, and G. Lachapelle, "Detection performance of polarization and spatial diversities for indoor GNSS applications," International Journal of Antennas and Propagation, vol. 2012, Article ID 879142, 10 pages, 2012. 
[17] E. D. Kaplan and C. J. Hegarty, Understanding GPS: Principles and Applications, Artech House, Boston, Mass, USA, 2006.

[18] E. L. Houghton and P. W. Carpenter, Aerodynamics for Engineering Students, Edward Arnold, London, UK, 4th edition, 1993.

[19] R. Clarke, "A statistical theory of mobile radio reception," Bell System Technical Journal, vol. 47, pp. 957-1000, 1968.

[20] M. A. Jensen and Y. Rahmat-Samii, "Performance analysis of antennas for hand-held transceivers using FDTD," IEEE Transactions on Antennas and Propagation, vol. 42, no. 8, pp. 1106-1112, 1994.

[21] T. Taga, "Analysis for mean effective gain of mobile antennas in land mobile radio environments," IEEE Transactions on Vehicular Technology, vol. 39, no. 2, pp. 117-131, 1990.

[22] P. Mattheijssen, M. H. A. J. Herben, G. Dolmans, and L. Leyten, "Antenna-pattern diversity versus space diversity for use at handhelds," IEEE Transactions on Vehicular Technology, vol. 53, no. 4, pp. 1035-1042, 2004.

[23] R. Vaughan, "Switched parasitic elements for antenna diversity," IEEE Transactions on Antennas and Propagation, vol. 47, no. 2, pp. 399-405, 1999.

[24] S. R. Saunders, Antennas and Propagation for Wireless Communication Systems, John Wiley \& Sons, New York, NY, USA, 1999.

[25] C.-L. Chang, H.-N. Shou, and J.-C. Juang, "Spatial cooperative diversity technique in performance enhancement of GNSS receivers," in Proceedings of the 8th Asian Control Conference (ASCC '11), pp. 1239-1244, May 2011.

[26] M. Tarkiainen and T. Westman, "Predictive switched diversity for slow speed mobile terminals," in Proceedings of the 47th IEEE Vehicular Technology Conference, vol. 3, pp. 2042-2044, May 1997.

[27] A. Sendonaris, E. Erkip, and B. Aazhang, "User cooperation diversity-part I and part II," IEEE Transactions on Communications, vol. 51, no. 11, pp. 1927-1948, 2003.

[28] T. S. Rappaport, Wireless Communications Principles and Practice, Pearson Education International, Upper Saddle River, NJ, USA, 2nd edition, 2002.

[29] C.-K. Park and K.-S. Min, "A study on spatial correlation characteristic of array antenna for multi antenna system," in Proceedings of the Asia-Pacific Microwave Conference (APMC '05), vol. 3, December 2005.

[30] W. C. Jakes, Microwave Mobile Communications, IEEE Press, Piscataway, NJ, USA, 1994.

[31] G. L. Stüber, Principles of Mobile Communications, Springer, New York, NY, USA, 2nd edition, 2000.

[32] S. M. Kay, Fundamentals of Statistical Signal Processing, Volume II: Detection Theory, Prentice Hall PTR, Upper Saddle River, NJ, USA, 1998.

[33] S. R. Saunders, Antennas and Propagation for Wireless Communication Systems, John Wiley \& Sons, New York, NY, USA, 2nd edition, 2007.

[34] I. E. Telatar, "Capacity of multi-antenna Gaussian channels," European Transactions on Telecommunications, vol. 10, no. 6, pp. 585-595, 1999. 


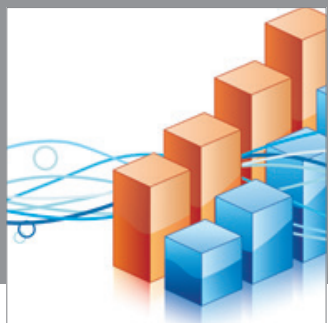

Advances in

Operations Research

mansans

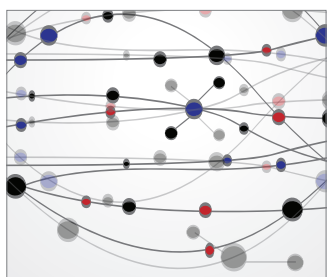

The Scientific World Journal
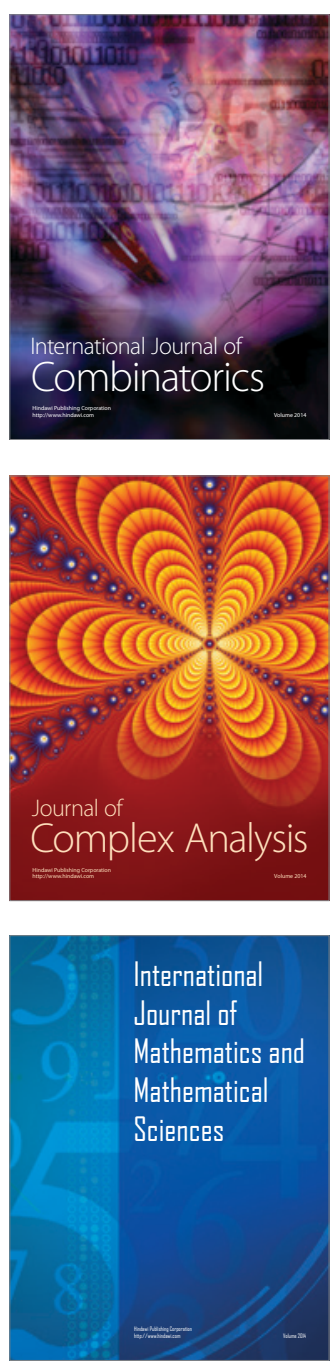
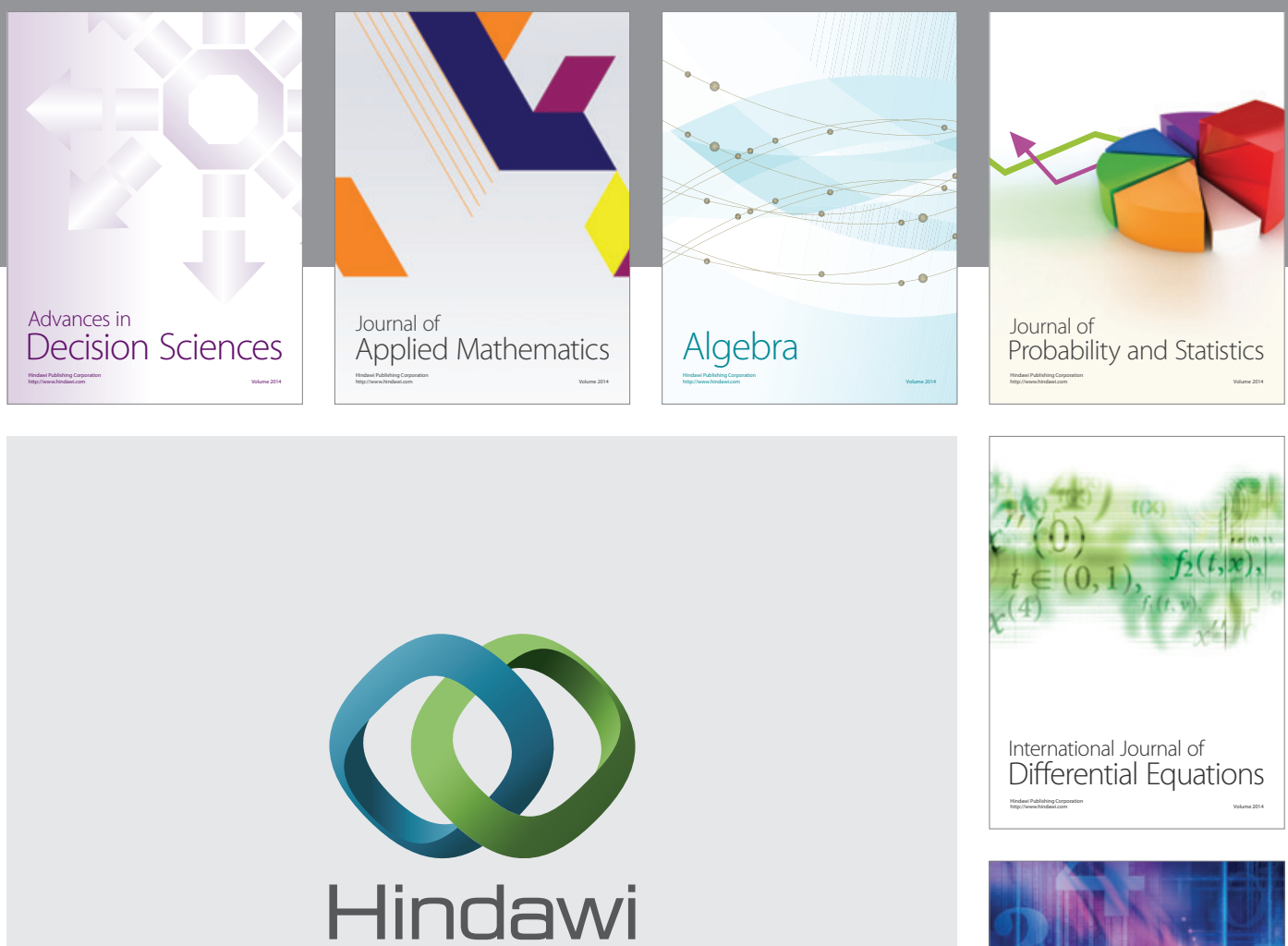

Submit your manuscripts at http://www.hindawi.com
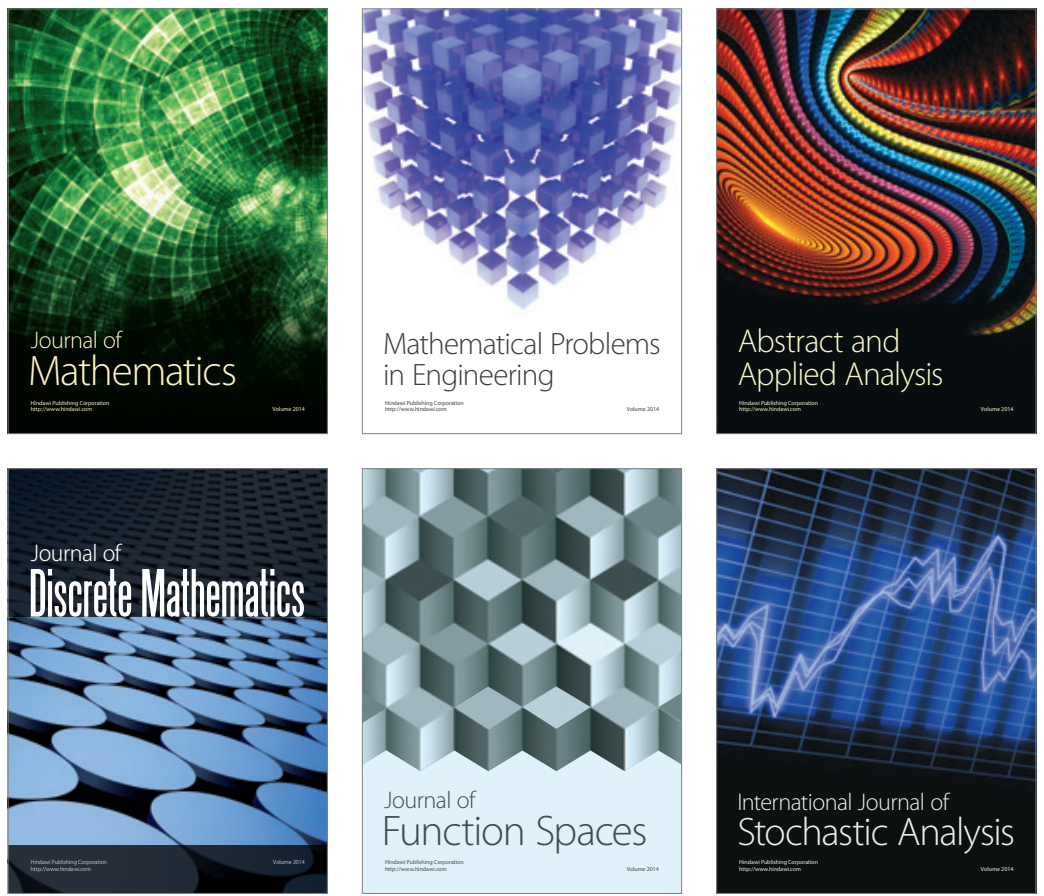

Journal of

Function Spaces

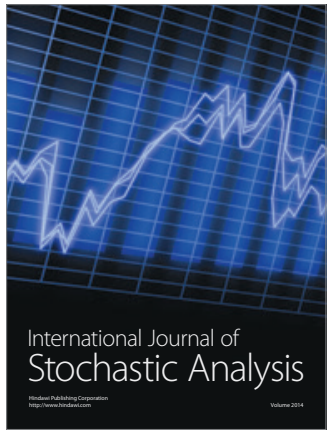

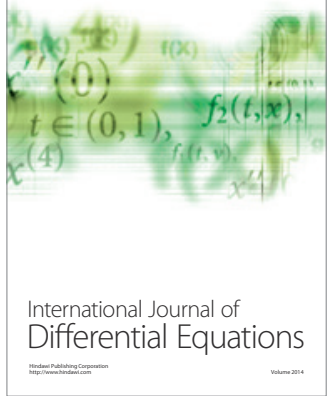
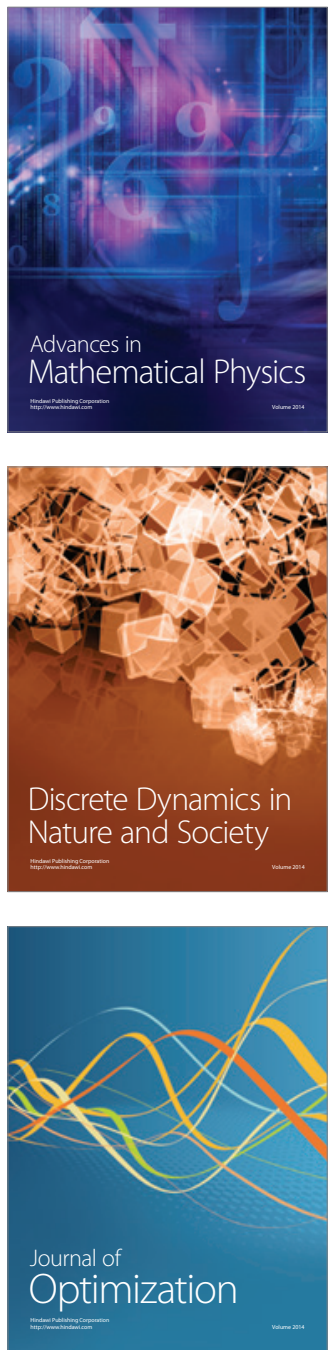